\title{
CORRESPONDENCE
}

\section{Value of exposure: External postings of plastic surgery trainees in}

\section{India}

\author{
Rahul Bamal ${ }^{*}$, Amrita Mandal \\ Department of Burns, Plastic \& Maxillofacial Surgery, VMMC \& Safdarjung Hospital, New Delhi, India
}

Citation: Bamal R and Mandal A. Value of exposure: External postings of plastic surgery trainees in India. J Surg Dermatol 2021; 6(1): 135; http://dx.doi.org/10.18282/jsd.v6.i1.135.

*Correspondence to: Rahul Bamal, Bamal Hospital, Rohtak, Haryana, India; rahulturbo@yahoo.co.uk

Received: $8^{\text {th }}$ October 2020; Published Online: $24^{\text {th }}$ October 2020

\section{Dear Editor,}

The medical education system in India is not perfect and recent developments have again raised questions about the working and conduct of the Medical Council of India. There are loud calls for standardization and, in the midst of this chaos, there are always people who strive for excellence, who try to absorb knowledge at every opportunity and who can burst onto the mega screen anytime. Our aim should be to provide them with the congenial environment in which they can flourish ${ }^{[1]}$.

While student exchanges and external postings are considered to be an integral part of surgical training all over the world, there are not many plastic surgery units regularly offering the same in India. The whole purpose is to inculcate innovative ideas and develop new perspectives to further our growth. We were fortunate to get this opportunity during the second year of our MCh Plastic \& Reconstructive Surgery training. Though our centre offers well-rounded exposure, there are still many specialized units in the country that are breaking barriers in various fields of plastic surgery and are also recognized internationally for it.

The first of our visits was to Ganga Hospital, Coimbatore, which was an eye opener of sorts as far as the importance of inter-disciplinary coordination between anaesthetists, orthopedicians and plastic surgeons are concerned. Anaesthetists here are the first to receive patients, practicing 'In Theatre Resuscitation', and are also responsible for bringing the patient to the operating table with surgeons doing their work alongside. 'Block on Arrival' protocol is followed, which makes the patient pain-free and compliant as soon as possible. The emphasis on thorough initial debridement drastically reduces complication rates ${ }^{[2]}$.

Consultants are always ready to teach, demonstrate, explain and make us understand. We learnt new techniques, observed new procedures and revised old ways. (Figures 1, 2A and 2B) depict some of the interesting clinical exposures that we obtained.

We attended a local monthly plastic surgery meeting and an APSI (Association of Plastic Surgeons of India)-accredited hand course, and visited a skin and bone bank (one of few in the country) during our stay.

After these three weeks of experience, we headed to another renowned centre, namely Specialist's Hospital, Kochi. Here, the work culture was more relaxed but with similarly excellent patient management and outcomes. We also observed blepharoplasties, brow lifts, abdominoplasties and breast reductions - the different procedures from what we had experienced at the previous centre and the other similar ones. The highlight of the stay was our last surgery, an upper thigh bilateral double replant after a train accident, which was unfortunately not successful, but taught us a lot including an innovative intra-operative cooling technique ${ }^{[3]}$. 
The whole experience was enriching, rewarding and truly satisfying. We went back kindled with the excitement of promising possibilities and seriously hope that this trend of external postings will not only continue, but expand to each and every unit and specialty of our country.

\section{Acknowledgements}

We thank Dr. Karoon Agrawal, Department of Burns, Plastic \& Maxillofacial Surgery, VMMC \& Safdarjung Hospital, New Delhi; Dr. S. Raja Sabapathy, Department of Plastic Surgery, Hand and Microsurgery and Burns, Ganga Hospital, Coimbatore; and Dr. R. Jayakumar, Specialist's Hospital, Kochi, for their support and encouragement.

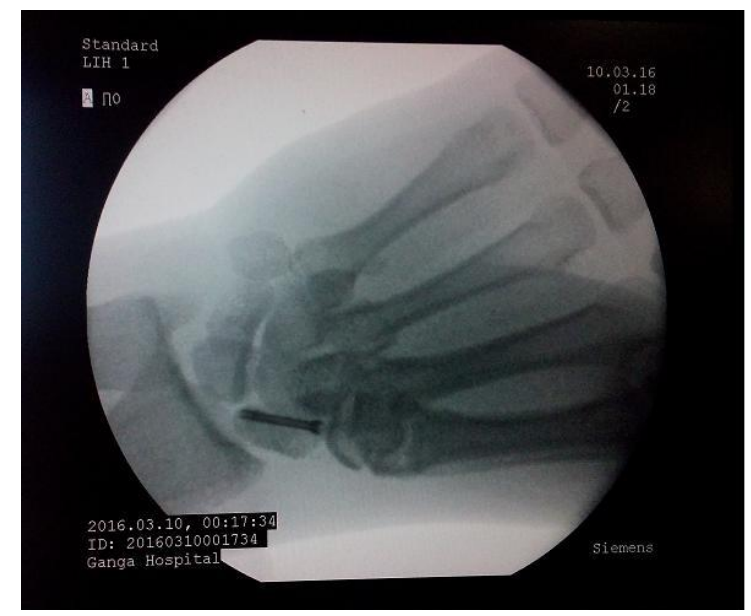

Figure 1. Closed reduction and Herbert's screw fixation of scaphoid fracture

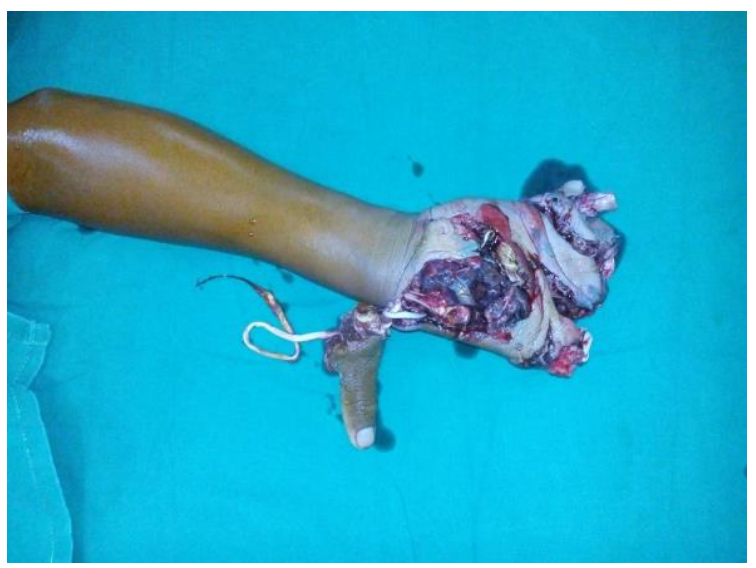

Figure 2A. Cracker blast injury right hand with thumb avulsion

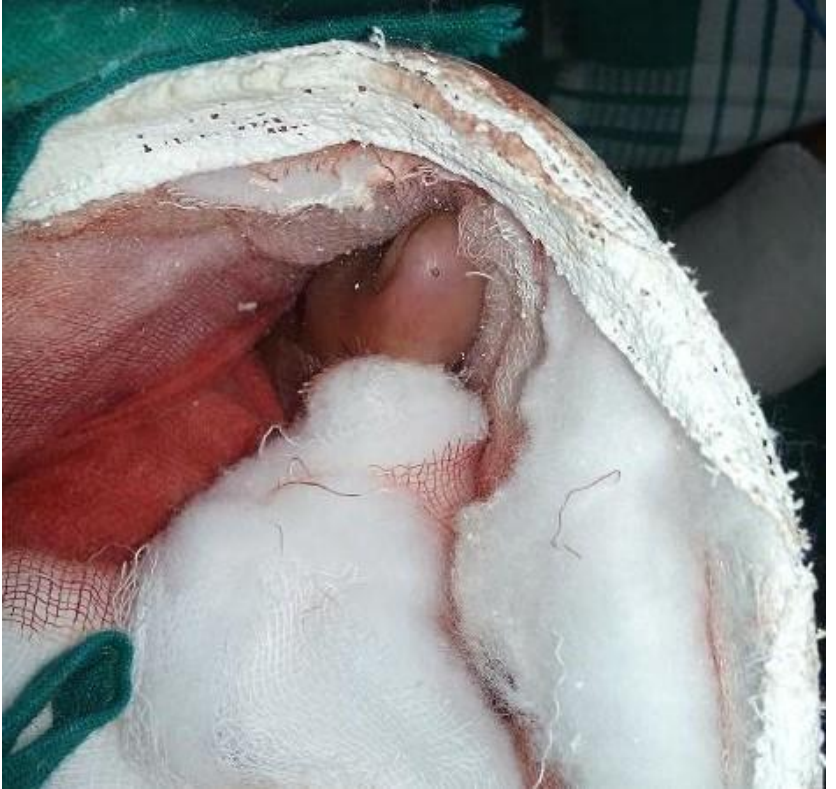

Figure 2B. Replanted thumb of same patient at 24 hourswarm and pink

\section{Conflict of interest}

The authors declare no potential conflict of interest with respect to the research, authorship, and/or publication of this article.

\section{References}

1. Parliament of India, Rajya Sabha. The functioning of Medical Council of India. Department-related parliamentary standing committee on health and family welfare [Internet]. Ninety-second report; 2016 [cited 2017 Feb 6]. Available from: http://164.100.47.5/ newcommittee/reports/EnglishCommittees/Committee $\% 20$ on $\% 20 \mathrm{He}$ alth\%20and\%20Family\%20Welfare/92.pdf.

2. Sabapathy SR, Venkatramani H, Bharathi RR, Dheenadhayalan J, Bhat VR, et al. Technical considerations and functional outcome of 22 major replantations (The BSSH Douglas Lamb Lecture, 2005). J Hand Surg Eur 2007; 32(5): 488-501. doi: 10.1016/J.JHSE.20 07.06.013.

3. Jayakumar R, Bamal R, Mandal A. A novel technique for continuous cooling till re-establishment of blood flow during major limb replants. Indian J Plast Surg 2016; 49(3): 429-430. doi: 10.4103/0970-0358.197222. 